\title{
Sistema de informações sobre nascidos vivos: um estudo de revisão
}

\section{Brazilian live birth information system: a review study}

$N$ atália Santana Paiva ${ }^{1}$

Claudia M edina Coeli ${ }^{2}$

Arlinda Barbosa M oreno ${ }^{1}$

Raphael M endonça Guimarães ${ }^{2}$

Kenneth Rochel deCamargo Júnior ${ }^{3}$

${ }^{1}$ Escola Politécnica de SaúdeJoaquim Venâncio, Fundação O swaldo Cruz. Av. Brasil 4.365,

M anguinhos. 21040-360

Rio deJaneiro RJ.

natalia_uff@hotmail.com

2Instituto de Estudosem

Saúde Coletiva,

UniversidadeFederal do

Rio deJaneiro.

${ }^{3}$ Instituto deM edicina

Social, Universidade do

Estado do Rio deJaneiro.
Abstract The aim of this study was to perform a systematic review of the use of the Live Birth Information System (Sistema de Informações de Nascidos Vivos - SINASC) in health research. MEDLINE, LILACS and SCIELO databases were searched from 1994 to 2005 using the following combination of descriptors: "SINASC", "livebirth", "Brazil". We identified 157 abstracts within the reference period, among which 44 were selected and classified according to specific criteria. The number of articles published per year increased duringtheperiod studied. Themajority of thestudies was carried out in the Southeast region and used the municipality as the geographic unity of analysis. A varied range of subjects were assessed including descriptions of livebirths profiles, health service and programs evaluations and risk factors for infant outcomes. In conclusion, although the SIN ASC represents an important data source for maternal-infant health research, the system's coverage and the reliability of its data need to be better evaluated.

Key words Information systems, Live births, Literature review
Resumo 0 objetivo desteestudo foi realizar uma revisão de literatura sobre o uso do Sistema de I nformações sobre $N$ ascidos Vivos (SINASC) na pesquisa em saúde. As bases de dados M ED LINE, LILACS e SciELO foram pesquisadas no período de 1994 a 2005, utilizando-se a combinação dos seguintes descritores: "SINASC", "live birth", "Brazil". Foram identificados 157 resumos dentro do período de referência, dos quais 44 foram selecionados e classificados segundo critérios específicos. 0 número de artigos publicados por ano cresceu ao longo do período estudado. A maioria dos estudos foi desenvolvida na região Sudeste e empregou o município como a unidade geográfica da análise. U ma gama variada de temas foi avaliada, incluindo descriç̧̃es do perfil de nascidos vivos, avaliações de serviços e programas de saúde e fatores de risco para desfechos infantis. Concluindo, embora o SINASC represente uma fonte de dados importantepara a pesqui sa sobrea saúde materno-infantil, a cobertura do sistema e a confiabilidade dos seus dados precisam ser meIhor avaliadas.

Palavras-chave Sistemas de informações, N ascidos vivos, Revisão de literatura 


\section{Introdução}

Em 1990, o Sistema de I nformações sobre N ascidos Vivos (SINASC) foi implantado pelo Ministério da Saúde visando ao registro sistemático, em âmbito nacional, de informações sobre os nascimentos vivos ${ }^{1-4}$.

O SINASC se baseia no instrumento Declaração de $N$ ascido $V$ ivo (DN), que contemplauma série de dados sobre a mãe, o pré-natal, o parto e o recém-nascido.

Esse sistema representa uma fonte de informação relevante para a pesquisa e avaliação em saúde na área materno-infantil. Em função disso, o mesmo tem sido crescentemente empregado como fonte de dados em publicações científicas. Apesar do acúmulo da produção bibliográfica relacionada ao SINASC, nenhum trabalho buscou, até o momento, sistematizar essa produção.

0 presente estudo tem por objetivo realizar uma revisão de literatura em bases de referências bibliográficas, no período de 1994 a 2005, sobre artigos que empregaram o SINASC como fonte de dados.

\section{Metodologia}

Foi realizada uma busca nas bases de referências bibliográficas M EDLINE, LILACS, SciELO enas referências dos artigos analisados, no período compreendido entre 1994 a 2005.

A estratégia de busca na base M EDLINE foi baseada na pesquisa, em todos os campos, da seguinte chave: "(SINASC OR live birth) AND (Brazil OR Brasil)". Para as demais bases, foram empregados os unitermos correspondentes em português e utilizada sintaxe correspondente específica da base.

As referências identificadas foram armazenadas e processadas por meio do programa ProCite ${ }^{\circledR}$. Foram selecionados os artigos que empregaram o SINASC como fonte de dados, tendo sido considerados artigos originais (delineamento experimental ou observacional) e excluídos editoriais, cartas, comentários, artigos de revisão ou meta-análise.

Essa busca resultou em 151 resumos (excluindo as duplicidades). Destes, 61 artigos não utilizaram o SINASC em momento algum, sendo excluídos. Os noventa artigos restantes foram obtidos na íntegra e, a seguir, analisados. Após a fase de leitura dos textos completos, foram excluídos outros 52 artigos por serem meta-análise, revisões sistemáticas e artigos quenão utiliza- ram o SINASC como fonte de dados, restando, até então, 38 artigos para fazer parte deste estudo. Posteriormente, as referências desses 38 já selecionados foram analisadas e seis delas foram adicionadas ao estudo por utilizarem o SINASC como fonte de dados.

A leitura desses artigos caracterizou- os segundo: (a) ano de publicação; (b) tipo de periódico (área de conhecimento e classificação Qualis da Coordenação de Aperfeiçoamento de Pessoal de Nível Superior - CAPES). Vale mencionar que o Qualis"é o resultado do processo de classificação dos veículos utilizados pelos programas de pósgraduação para a divulgação da produção intelectual de seus docentes ealunos"6; (c) características das instituições executoras dos estudos (ensino/pesquisa ou assistência/gestão, região de localização); (d) população de estudo (período, abrangência geográfica, região analisada); e (e) tipos de delineamento dos estudos. Além disso, os mesmos foram agrupados em seis vertentes, desenhadas a partir da adaptação do estudo de Bittencourt et al. ${ }^{7}$ : (1) qualidade da informação; (2) estratégias para potencializar o uso das informações para gestão, pesquisa e assistência médico-hospitalar; (3) descrição do perfil denascimento; (4) vigilância da mortalidade materna; (5) políticas de programas e serviços de saúde; (6) avaliação da associação entrefatores distais e proximais e desfechos na área materno-infantil.

\section{Resultados}

$\mathrm{Na}$ década de noventa, foram publicados catorze artigos (31,8\%), sendo o primeiro em 1996. Nessemesmo período, merece destaque 0 ano de 1997, no qual foram publicados oito artigos (18,2\%) (Tabela 1).

A partir de 2000, trinta artigos (68,2\%) foram publicados, merecendo destaque os anos $2001 \mathrm{e}$ 2005, queal cançaram oito enove publicações, respectivamente (18,2\% e 20,5\%) (Tabela 1 ).

Em relação ao tipo de periódico, háu uma gama de artigos indexados em veículos da área de saúde coletiva ( $86,4 \%$ ) e 32 deles $(72,7 \%$ ) foram classificados como A Internacional, segundo a CAPES (Tabela 1).

Quanto à execução dos trabalhos, 112 autores estão envolvidos com a produção dos estudos e estes, por sua vez, estão vinculados a dezenove instituições de "ensino epesquisa" (55,9\%), doze de "assistência e gestão" (35,3\%) e três internacionais provenientes do Reino Unido (8,8\%) (Tabela 2). 
Tabela 1. Perfil dos estudos que empregam o SINASC como fonte de dados, segundo ano de publicação e tipo de periódico.

\begin{tabular}{|c|c|c|c|}
\hline & $\mathrm{N}^{(1)}$ & $\%$ & Referências bibliográficas \\
\hline \multicolumn{4}{|l|}{ Ano de publicação } \\
\hline 1996 & 2 & 4,5 & 8,20 \\
\hline 1997 & 8 & 18,2 & $13,15,21,22,23,26,27,34$ \\
\hline 1998 & 3 & 6,8 & $16,24,46$ \\
\hline 1999 & 1 & 2,3 & 28 \\
\hline 2000 & 2 & 4,5 & 38,47 \\
\hline 2001 & 8 & 18,2 & $11,14,17,18,29,40,41,51$ \\
\hline 2002 & 5 & 11,4 & $12,37,42,43,53$ \\
\hline 2003 & 4 & 9,1 & $30,33,35,52$ \\
\hline 2004 & 2 & 4,5 & 10,45 \\
\hline 2005 & 9 & 20,5 & $9,19,25,36,39,44,48,49,50$ \\
\hline \multicolumn{4}{|l|}{ Periódico } \\
\hline \multicolumn{4}{|l|}{ Área } \\
\hline Saúde coletiva & 38 & 86,4 & $\begin{array}{l}8,9,10,11,12,13,14,15,16,17,18, \\
19,20,21,22,23,24,26,27,28,33, \\
34,35,36,37,38,39,41,42,43,44, \\
46,47,48,50,51,52,53\end{array}$ \\
\hline Outras & 6 & 13,6 & $25,29,30,40,45,49$ \\
\hline \multicolumn{4}{|c|}{ Circulação Qualis Saúde coletiva } \\
\hline A Internacional & 32 & 72,7 & $\begin{array}{l}9,10,11,12,16,17,18,19,20,21 \\
22,26,28,33,34,35,36,37,38,39, \\
41,42,43,44,45,46,47,48,50,51 \\
52,53\end{array}$ \\
\hline B Nacional & 7 & 15,9 & $8,13,14,15,23,24,27$ \\
\hline C Internacional & 4 & 9,1 & $25,29,30,40$ \\
\hline A Nacional & 1 & 2,3 & 49 \\
\hline
\end{tabular}

Doze dos 44 artigos foram executados por meio de parcerias entre instituições de "ensino e pesquisa" com as de "assistência e gestão" (27,3\%). E dez $(22,7 \%)$ foram realizados sem parcerias entre instituições, mas, em alguns casos, havendo parcerias entre departamentos das mesmas (Tabela 2).

Com relação à localização das instituições, a maioria delas está concentrada na região Sudeste $(44,1 \%)$ seguida pelo Nordeste(23,5\%) (Tabela2).

Quanto ao perfil dos estudos selecionados, a maioria deles $(52,3 \%$ ) foi realizada no período inicial de utilização maisabrangentedo SINASC (19952000) (Tabela 3). Além disso, o delineamento seccional foi o mais empregado (43,2\%), seguido pelo ecológico (38,6\%) e coorte (18,2\%) (Tabela 3).

Em relação ao agrupamento nas seis vertentes mencionadas, seteartigos ( $15,9 \%$ ) abordaram a qualidadeea confiabilidade da informação (vertente 1). $\mathrm{Na}$ segunda vertente, estratégias para potencializar o uso das informações para gestão, pesquisa e assistência médico-hospitalar, seis estudos $(13,6 \%)$ foram localizados. Dez estudos $(22,7 \%)$ foram classificados como descrição do perfil dos nascimentos (vertente 3 ). Q uanto à vigilância da mortal idadematerna (vertente4), não houve nenhum estudo que atendesse a esse delineamento; sete estudos foram alocados em "políticas de programas e serviços de saúde" (vertente 5) e, por fim, catorze deles $(31,2 \%)$ foram classificados na vertente 6 (associação entre fatores e proximais e distais e desfechos na área maternoinfantil). Nestaúltima vertente, os desfechos mais avaliados foram a mortalidade infantil, a gestação em adolescentes, o baixo peso ao nascer, a prematuridade e o parto cesáreo (Tabela 3).

Os estudos tiveram lugar em 45 locais distintos de análise, sendo apenas cinco estudos relacionados ao Brasil como um todo (11,1\%). Apenas um estudo se deu na região $\mathrm{N}$ orte $(2,2 \%)$ ea maior concentração de trabalhos ocorreu na re gião Sudeste (51,1\%) (Tabela 3). 


\begin{tabular}{|c|c|c|c|c|}
\hline \multicolumn{5}{|c|}{ Tabela 2. Perfil das instituições executoras dos estudos. } \\
\hline & & $\mathrm{N}$ & $\%$ & Referências bibliográficas \\
\hline & \multicolumn{4}{|l|}{ Unidade $^{(1)}$} \\
\hline & Ensino e pesquisa & 19 & 55,9 & $\begin{array}{l}8,9,10,11,12,14,16,17,18,19,20, \\
21,24,26,27,28,30,33,34,35,37,38, \\
39,40,41,42,43,44,45,46,47,48,49, \\
50,51,52,53\end{array}$ \\
\hline & Assistência e gestão & 12 & 35,3 & $\begin{array}{l}8,9,10,13,15,17,18,22,23,25,29 \\
30,33,35,36,38,41,46,48,50\end{array}$ \\
\hline & \multicolumn{4}{|l|}{ Parceria ${ }^{(2)}$} \\
\hline & Sem parceria & 10 & 22,7 & $14,15,16,20,25,26,27,29,40,42$ \\
\hline & Instituição de ensino e pesquisa & 15 & 34,1 & $11,12,19,21,24,28,34,37,39,43,44$ \\
\hline & Instituição de assistência e gestão & 4 & 9,1 & $47,49,52,53$ \\
\hline & $\begin{array}{l}\text { Instituição de ensino e pesquisa \& } \\
\text { assistência e gestão }\end{array}$ & 12 & 27,3 & $13,22,23,36$ \\
\hline & Internacional & 3 & 6,8 & $\begin{array}{l}8,9,10,17,18,30,33,35,38,41,46,48 \\
45,50,51\end{array}$ \\
\hline & \multicolumn{4}{|r|}{ (1) } \\
\hline & $\begin{array}{l}\text { SE } \\
N E \\
S\end{array}$ & 15 & 44,1 & $\begin{array}{l}8,9,10,12,14,16,17,18,20,21,22 \\
23,26,27,28,29,30,34,35,36,37,38 \\
39,41,43,44,45,46,47,49,51,52,53\end{array}$ \\
\hline & Centro-Oeste & 8 & 23,5 & $11,19,25,28,33,40,42,52,53$ \\
\hline & Internacional & 5 & 14,7 & $24,50,51,53$ \\
\hline & & 3 & 8,8 & $8,13,15,17,39,47,48,49$ \\
\hline & & 3 & 8,8 & $45,50,51$ \\
\hline
\end{tabular}

(1) $\mathrm{N}=34$; $^{\text {(2) }} \mathrm{N}=44$.

Q uanto à abrangência da unidade geográfica do estudo, houve predominância de estudos que empregaram o município (70,5\%), sendo observados cinco estudos de abrangência nacional $(11,4 \%)$, quatro estaduais $(9,1 \%)$, dois macrorregionais $(4,5 \%)$ e, por fim, doisutilizaram bairros ou distritos $(4,5 \%)$ (Tabela 3$)$.

\section{Discussão}

N esta revisão de literatura, observamos um crescimento do número de publicações em períodos mais recentes. Embora o SINASC tenha sido implantado em 1990, o acesso amplo as suas bases de dados foi possível somente em anos mais recentes, inicialmente por meio de CD-ROM e, posteriormente(1994), no sitedo DATASUS. Este fato, juntamente com a popularização do uso desse sistema para fins de avaliação e pesquisa, pode explicar o padrão temporal observado ${ }^{1-4}$.

Da mesma forma que 0 verificado por Bittencourt et al. ${ }^{7}$, que realizaram uma revisão sobre o uso do Sistema de Informações H ospitala- res do Sistema Único de Saúde (SIH -SUS), há uma concentração geográfica de autores filiados a instituições localizadas na região Sudeste, bem como de estudos analisando os dados provenientes da mesma. A maioria dos estudos foi realizada por autores filiados a instituições de ensino e pesquisa, havendo, entretanto, um crescimento mais recente de artigos elaborados por autores ligados a instituições de assistência e gestão. Este achado pode estar refletindo o reconhecimento do SINASC como fonte de dados relevante para a avaliação de programas e serviços de saúde. 0 envolvimento desses profissionais na execução dos estudos é essencial, já que as instituições de assistência e gestão são responsáveis pela produção e garantia da qualidade dos dados do SINASC.

Observa-se o predomínio de artigos publicados em periódicos classificados como A Internacional pelo sistema Qualis (CAPES); isso se dá em função da maior proporção de artigos publicados nos periódicos Cadernos de Saúde Publica e Revista de Saúde Pública. Chama atenção, ainda, o reduzido número de artigos publicados em 


\begin{tabular}{|c|c|c|c|}
\hline & $\mathrm{N}$ & $\%$ & Referências bibliográficas \\
\hline \multicolumn{4}{|l|}{ Período(1) } \\
\hline 1990-1994 & 19 & 43,2 & $\begin{array}{l}8,13,16,17,18,20,21,22,23,24,25,27,28,34,36,39,46 \\
47,51\end{array}$ \\
\hline $1995-2000$ & 23 & 52,3 & $\begin{array}{l}9,10,11,12,14,15,19,26,29,30,35,37,38,40,41,42,43 \\
44,45,48,50,52,53\end{array}$ \\
\hline 2001-2005 & 2 & 4,5 & 33,49 \\
\hline \multicolumn{4}{|l|}{ Tipo } \\
\hline Seccional & 19 & 43,2 & $\begin{array}{l}10,11,14,22,23,24,26,27,29,30,33,40,41,42,43,44,45 \text {, } \\
46,50\end{array}$ \\
\hline Ecológico & 17 & 38,6 & $8,9,12,13,15,16,17,19,20,21,25,36,37,38,51,52,53$ \\
\hline Coorte & 8 & 18,2 & $18,28,34,35,39,47,48,49$ \\
\hline \multicolumn{4}{|l|}{ Vertentes ${ }^{(1),(3)}$} \\
\hline 1 & 7 & 13,6 & $8,9,10,11,12,13,14$ \\
\hline 2 & 6 & 13,6 & $15,16,17,18,19,20$ \\
\hline 3 & 10 & 22,7 & $21,22,23,24,25,26,27,28,29,30$ \\
\hline 5 & 7 & 15,9 & $33,34,35,36,37,38,39$ \\
\hline 6 & 14 & 34,1 & $40,41,42,43,44,45,46,47,48,49,50,51,52,53$ \\
\hline \multicolumn{4}{|r|}{ 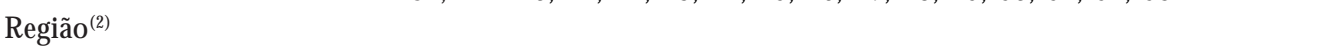 } \\
\hline SE & 23 & 51,1 & $\begin{array}{l}10,16,18,20,21,22,23,26,27,28,29,30,34,35,36,37,38 \text {, } \\
41,43,44,45,46,51\end{array}$ \\
\hline $\mathrm{NE}$ & 8 & 17,8 & $11,14,19,25,33,40,42,52$ \\
\hline Centro-O este & 5 & 11,1 & $17,39,47,48,49$ \\
\hline Brasil & 5 & 11,1 & $8,9,12,13,15$ \\
\hline S & 3 & 6,7 & $24,50,53$ \\
\hline $\mathrm{N}$ & 1 & 2,2 & 14 \\
\hline \multicolumn{4}{|l|}{ Abrangência(1) } \\
\hline M unicipal & 31 & 70,5 & $\begin{array}{l}10,11,16,17,18,19,20,21,22,24,26,27,29,30,34,35,36, \\
38,39,40,41,42,43,44,46,47,48,49,51,53,52\end{array}$ \\
\hline Nacional & 5 & 11,4 & $8,9,12,13,15$ \\
\hline Estadual & 4 & 9,1 & $23,28,37,45$ \\
\hline M acrorregião & 2 & 4,5 & 14,50 \\
\hline Bairros ou distritos & 2 & 4,5 & 25,33 \\
\hline
\end{tabular}

(1) $\mathrm{N}=44 ;{ }^{(2)} \mathrm{N}=45 ;{ }^{(3)}$ Vertentes: 1 - Qualidade da informação; 2 - Estratégias para potencializar o uso das informações para gestão, pesquisa eassistência médico-hospitalar; 3 - Descrição do perfil de nascimento; 5 - Políticas de programas e serviços de saúde; 6 - Avaliação da associação entre fatores distais e proximais e desfechos na área materno-infantil.

periódicos de áreas mais clínicas, o que sugere um menor conhecimento sobre o potencial do SINASC para fins de investigação entre os pesquisadores dessas áreas.

Diferentemente do observado na revisão sobre o SIH-SUS ${ }^{7}$, as unidades de análise geográfica mais frequentemente empregadas nos artigos que utilizaram o SINASC, são as menores unidades (por exemplo, municípios), sendo mais raros os estudos que abordaram macrorregiões e o Brasil como um todo.

Com relação aos delineamentos de estudo empregados, o de coortevem sendo utilizado em estudos mais recentes, em geral, por meio do emprego de técnicas de linkage das bases de da- dos do SINASC com as bases do SIH-SUS e, especialmente, com as bases de dados do Sistema de Informações sobre Mortalidade (SIM). Tal fato pode ser devido, também, ao maior desenvolvimento e disponibilização de tecnologias de integração de bases de dados e de equipamentos de informática (hardwares e softwares).

Na primeira vertente, qualidade da informação, foram realizados sete estudos a partir dos dados do SINASC e do SIM para mortalidade maternae infantil.

M ello Jorge et al. ${ }^{8}$ compararam os dados do SINASC com outros sistemas de informação como o SIH -SUS e o Sistema de Informação da Atenção Básica (SIAB) e ainda com outras fon- 
tes primárias de dados. Já Cardoso et al. ${ }^{9}$ analisaram a consistência dos Sistemas de Informações sobre M ortalidade (SIM) e sobre Nascidos Vivos (SIN ASC) como fontes de dados para a avaliação de desigualdades em raça/cor em saúde no Brasil. Em seguida, Theme Filha et al. ${ }^{10}$ avaliaram a cobertura e a confiabilidade das variáveis constantes no Sistema de Informações sobre Nascidos Vivos, no município do Rio de Janeiro, discutindo a importância da qualidade das informações do SINASC e o seu uso como instrumento de definição de políticas na área materno-infantil. Silva et al. ${ }^{11}$ verificaram a concordância entre as informações constantes no Sistema de Informação sobre $\mathrm{N}$ ascidos Vivos (SIN ASC) referentes a partos hospitalares eaquelas obtidas por inquérito seccional (padrãoouro). A análise do arquivo do SINASC mostrou que a cobertura estimada foi baixa; a taxa de baixo peso ao nascer pareceter boa validade e reprodutibilidade; ea taxa de prematuridadeestá subestimada. Szwarcwald et al. ${ }^{12}$ apresentaram uma proposta metodológica para a estimação da mortalidade infantil, no Brasil, mediante a utilização das informações do M inistério da Saúde, que consistiu em estabelecer critérios para identificar municípios com limitações nas informações, propondo um índice para sintetizar a sua adequação. Oliveira e Pereira ${ }^{13}$ observaram os principais problemas que ocorrem com os dados de mortalidade e nascidos vivos, descrevendo-os e procurando uma explicação para os mesmos, aventando soluções para a minimização dos fatores que reduzem a qualidade equantidade dos dados; ao passo que M ello Jorge e Gotlieb ${ }^{14}$ compararam os totais dos dados de 1998, informados pelo SIN ASC, SIM e SI AB.

Há, a partir destes estudos, cobertura e avaliação dos dados do SINASC desde 1996 até 2002. Um problema detectado por todos os estudos, nesta vertente, refere-se ao elevado número de dados incompletos nos registros de nascidos vivos. Destaque-se quefoi aí alocado artigo da primeira avaliação dos dados brasileiros oriundos do SINASC ${ }^{8}$. Todavia, ainda há a necessidade de artigos mais recentes abordando a cobertura, qualidade e confiabilidade dos dados do sistema.

N a segunda vertente, estão as publicações cujo foco são as estratégias para potencializar o uso das informações para gestão, pesquisa e assistência médico-hospitalar. 0 primeiro deles ${ }^{15}$ discute a situação dos principais sistemas de informação gerenciados pelo M inistério da Saúde, dentre os quais o SINASC e o SIM. De uma forma geral, descreve o fluxo de informações, as variáveis, os indicadores relativos e as vantagens e limitações dos sistemas de informação. Sobreo SI M , destaca-se sua universalidade e o padrão de treinamento dos agentes, uniformizando os dados. Problemas apontados dizem respeito ao aumento do número de óbitos por causas mal definidas, preenchimento inadequado defichas e o retardo no processamento da informação, dificultando seu uso para a vigilância epidemiológica. Sobre o SINASC, a mesma universalidade do SIM é registrada, com a garantia, ainda, de variedade e propriedade das informações (gestação, parto e nascido vivo). D estaca-se, porém, como limitação, a subnotificação e a defasagem no processamento de informação.

Os demais estudos desta vertente se destacam pela utilização da análise espacial ${ }^{16-19}$ etécnicas de relacionamento probabilístico de dados ${ }^{20}$. A primeira mostrou-se uma metodologia capaz de aprofundar o conhecimento sobre o perfil de municípios para direcionar ações voltadas para áreas específicas. Já o linkage, que possui baixo custo operacional, mostrou-se viável e capaz de dimensionar adequadamente o perfil de assistência pré-natal e ao parto.

$\mathrm{Na}$ terceira vertente, estão estudos que descrevem o perfil de nascimento. Alguns dos estudos trabal ham dados de séries históricas ${ }^{21-25}$, enquanto outros utilizam dados para descrições pontuais ${ }^{26-30}$. Dois deles ${ }^{21,28}$ avaliam não o perfil dos nascidos vivos, mas sim, especificamente, a mortalidade infantil. N estes estudos, demonstrou-se que o uso contínuo dos sistemas de informação pelo nível municipal contribui para a diminuição da taxa de mortalidade infantil, especialmente quando são utilizadas técnicas de análise sobrepostas, como análise de causas múltiplas e distribuição geográfica²8.

Os estudos ainda caracterizam os nascidos vivos segundo via de parto, indicadores socioe conômicos da mãe e incidência de eventos como baixo peso ao nascer 22,24-27,29,30.

$\mathrm{N}$ ão foram localizados artigos empregando o uso do SINASC para a vigilância da morbimortalidade materna (vertente 4), ao contrário do observado para o SIH-SUS. Todavia, em buscas adicionais à literatura, foram local izadas uma dissertação de mestrado e uma tese de doutorado sobre esse tema, empregando o linkage probabilístico das bases do SINASC com as bases de dados do SIH-SUS e do SIM ${ }^{31,32}$, recentemente desenvolvidas.

$\mathrm{N}$ a quinta vertente, estão os artigos que avaliaram políticas de programas e serviços de saúde. Alguns dos estudos realizaram a avaliação da as- 
sistência prénatal por análise descritiva ${ }^{33,34}$. Destaca-se o estudo de Gentile et al. ${ }^{34}$, que revisa a hipótese deCarlos Gentile de M elo, na qual a forma de ressarcimento financeiro ao equipamento desaúde está relacionada com o aumento na taxa de cesáreas. 0 estudo sugere que a forma de re muneração não determina isoladamente 0 aumento da prevalência de cesáreas, mas há outras variáveis influenciando o fenômeno, como riscos médicos, fatores socioculturais e a esterilização cirúrgica. Há, ainda, outros estudos desenvolvidos a partir da análise de séries históricas ${ }^{35,36}$.

Um estudo de avaliação de um conjunto de indicadores da rededehospitais que prestam atendimento obstétrico ao SUS a partir do SIH-SUSe do $\mathrm{SINASC}^{37}$ foi realizado por meio da análise fatorial, pelo método de componentes principais. Além disso, um artigo para análise de assistência ao parto, para avaliar o perfil da clientela e o fluxo de demanda, utilizou técnica de mapeamento de fluxo ${ }^{38}$. Finalmente, há um estudo de coorte histórica constituído por meio do linkage do SIM e do SINASC que avaliou a associação entre via de parto e risco para morte neonatal ${ }^{39}$.

$\mathrm{Na}$ sexta vertente, sobre avaliação da associação entrefatores distais e proximais e desfechos na área materno-infantil, os artigos buscaram associações entre idade materna e vários desfechos (mortalidade infantil, prematuridade, baixo peso ao nascer, parto cesáreo e gestação em adolescentes quando associados a fatores proximais como poluição do ar, idade materna, realização de consultas pré-natal, entre outros). Alguns desses estudos, que abordaram os fatores socioeconômicos, versam, basicamente, sobre sua relação com a mortalidade infantil ou das taxas de cesáreas. A gravidez na adolescência foi associada a baixo peso ao nascer e prematuridade, bem como aponta uma série de fatores distais, como baixa idade materna, baixo status socioeconômico, número de consultas pré-natal, tabagismo e uso de drogas ilícitas ${ }^{40-44}$. Um estudo, ainda, procurou obter a associação entre a poluição do ar ambiente e o peso ao nascer, que demonstrou dose-resposta entre baixo peso ao nascer e concentração de monóxido de carbono no $\mathrm{ar}^{45}$. Costa eGotlieb ${ }^{46}$ desenvolveram um survey cujas associações estatisticamente significativas encontradas para baixo peso ao nascer fo- ram sexo feminino, prematuridade, mãe adolescente, mãe idosa e paridade maternal. Um conjunto de artigos nesta vertente ${ }^{47-49}$, pertencente a um mesmo programa de investigação em Goiânia avaliou, pelo método de linkage, o SIM e o SIN ASC, apontando uma associação entre mortalidade neonatal, baixo peso ao nascer, baixo Apgar 5 e prematuridade. Finalmente, Freitas et al. ${ }^{50}$ investigaram 0 efeito das desigualdades sociais nas taxas de cesarianas em primíparas com gravidez única em parto hospitalar e encontraram associação entre aumento de taxa de cesáreas com fatores sociais, econômicos e culturais, como escolaridade materna, raça, número de consultas pré natal e idade materna.

Destaca-se a ênfase dos artigos nos estudos das desigual dades socioeconômicas e das condições de vida. Goldani et al. ${ }^{51}$ determinaram as tendências da mortalidadeinfantil de 1995 a 1999, segundo a escolaridade materna, medidas em base geográfica, em Porto Alegre, apontando a redução nos componentes de mortalidade neonatal e pós-neonatal em área de mais baixa escolaridade materna. Já Guimarães et al. ${ }^{52}$ detectaram uma relação inversa entrea condi ção devida dos estratos e a magnitude da mortalidade infantil por grupo etário e causa básica, revelando desigualdades ocultas nos indicadores médios da cidade. Finalmente, Goldani et al. ${ }^{53}$ avaliaram a distribuição da mortalidade infantil, segundo uma classificação geoeconômica urbana, usando dados coletados rotineiramenteem nível municipal, avaliando que o monitoramento das desigualdades em saúde, por meio de dados de sistemas municipais de informação, pode ser progressivamente útil, dada a contínua descentralização do gerenciamento da saúde para o nível municipal no Brasil.

\section{Considerações finais}

O uso crescente do SINASC, abrangendo uma gama variada de temas nas áreas clínicas e da saúde coletiva, aponta a relevância do sistema como fonte de dados para a pesquisa e avaliação em saúde. Entretanto, é necessário que cresça o número de publicações de estudos que abordem a qualidade dos dados do sistema. 


\section{Colaboradores}

NS Paiva e CM Coeli são responsáveis pela concepção, delineamento, análise einterpretação dos dados e redação inicial do estudo. AB Moreno e KR Camargo Jr. colaboraram na análise einterpre tação dos dados e realizaram a revisão crítica do estudo. RM Guimarães colaborou na interpretação dos dados e na redação inicial do estudo.

\section{Referências}

1. Brasil. M inistério da Saúde. M anual de procedimentos do sistema de informações sobre nascidos vivos. Brasília: M inistério da Saúde; 2001.

2. Brasil. M inistério da Saúde. Portal da Saúde: SINASC. [site da Internet] [acessado 2006 nov 21]. Disponível em: http://portal.saude.gov.br/portal/ svs/visualizar_texto.cfm?idtxt=2132339

3. Camargo Jr $\overline{\mathrm{KR}}$, Coeli $\mathrm{CM}$. Políticas de Informação em Saúde. In: Escola Politécnica de Saúde Joaquim Venâncio. Texto de Apoio em Políticas de Saúde. $1^{a}$ ed. Rio de Janeiro: Fiocruz; 2005. p. 143-156.

4. Carvalho DM. Grandes sistemas nacionais de informação em saúde. Inf Epidemiol SUS 1997; 4:7-46.

5. ProCite 5 for Windows 1999. [site da Internet] [acessado 2008 mai 07]. Disponível em: http://www. procite.com/pcspecs.asp

6. Coordenação de Aperfeiçoamento de Pessoal de Nível Superior. Classificação Qualis. [site da Internet] [acessado 2008 mai 06]. Disponível em: http:/ /qualis.capes.gov.br/webqualis/

7. Bittencourt SA, Camacho LAB, Leal MC. O Sistema de Informação Hospitalar e sua Aplicação na Saúde Coletiva. Cad Saude Publica 2006; 22(1):19-30.

8. Mello Jorge M HP, Gotlieb SLD, Oliveira H. O Sistema de Informação sobre Nascidos Vivos: primeira avaliação dos dados brasileiros. Inf Epidemiol SUS 1996; 2:15-48.

9. Cardoso AM, Santos RV, Coimbra Jr. CEAA. Mortalidade infantil segundo raça/cor no Brasil: o que dizem os sistemas nacionais de informação? Cad Saude Publica 2005; 21(5):1602-1608.

10. Theme Filha MM, Gama SGN, Cunha CB, Leal MC. Confiabilidade do Sistema de Informações sobre $\mathrm{N}$ ascidos vivos $\mathrm{H}$ ospitalares no município do Rio de Janeiro, 1999-2001. Cad Saude Publica 2004; 20(Supl. 1):S83-S91.

11. Silva AAM, Ribeiro VS, Borba Jr. AF, Coimbra LC, Silva RA. Avaliação da qualidade dos dados do Sistema de Informações sobre Nascidos Vivos em 1997-1998. Rev. Saude Publica 2001; 35(6):508-514.

12. Szwarcwald CL, Leal MC, Andrade CLT, Souza Jr. PRB. Estimação da mortalidade infantil no Brasil: 0 que dizem as informações sobre óbitos e nascimentos do Ministério da Saúde? Cad Saude Publica 2002; 18(6):1725-1736.

13. Oliveira H, Pereira IPA. Estatísticas de Mortalidade e $\mathrm{N}$ ascidos Vivos: considerações sobre principais problemas. Inf Epidemiol SUS 1997; 3:15-19.

14. Mello Jorge M HP, Gotlieb SLD. O Sistema de Informação de Atenção Básica como Fonte de Dados para os Sistemas de Informações sobre M ortalidade e sobre Nascidos Vivos. Inf Epidemiol SUS 2001; 10(1):7-18.

15. Carvalho DM. Grandes sistemas nacionais de informação em saúde. Inf Epidemiol SUS 1997; 4:7-46.

16. d'Orsi E, Carvalho MS. Perfil de nascimentos no Município do Rio de Janeiro: uma análise espacial. Cad Saude Publica 1998; 14(2):367-379. 
17. Morais Neto OL, Barros M BA, Martelli CMT, Silva SA, Cavenaghi SM, Siqueira Jr. JB. Diferenças no padrão de ocorrência da mortalidade neonatal e pós-neonatal no M unicípio de Goiânia, Brasil, 19921996: análise espacial para identificação das áreas de risco. Cad Saude Publica 2001; 17(5):1241-1250.

18. Malta DC, Almeida MCM, Dias MAS, M erhy EE. A mortalidade infantil em Belo Horizonte, $M$ inas Gerais, Brasil, por área de abrangência dos Centros de Saúde (1994-1996). Cad Saude Publica 2001; 17(5):1189-1198.

19. Gurgel RQ, Dias IM O, França VLA, Castañeda DFN. Distribuição espacial do baixo peso ao nascer em Sergipe, Brasil, 1995/1998. Cad Saude Publica 2005; 21(5):1329-1337.

20. Almeida M F, M ello Jorge MHP. O uso da técnica Linkage de sistemas de informação em estudos de coorte sobre mortalidade neonatal. Rev. Saude Publica 1996; 30(2):141-147.

21. Gomes JO, Santo AH. Mortalidade infantil em municípios da região Centro-O este Paulista, Brasil, 1990 a 1992. Rev. Saude Publica 1997; 31(4):330-341.

22. Rodrigues CS, Magalhães Jr. HM, Evangelista PA, Ladeira RM, Laudares S. Perfil dos nascidos vivos no Município de Belo Horizonte, 1992-1994. Cad Saude Publica 1997; 13(1):53-57.

23. Silva RI, Theme Filha M M, Noronha CP. Sistema de Informação sobre nascidos vivos na cidade do Rio de Janeiro 1993/1996. Inf Epidemiol SUS 1997; 6(2):33-48.

24. Santa H elena ET, Wisbeck J. I mplantação do SINASC e perfil dos nascidos vivos de Blumenau, 1994-1997. Inf Epidemiol SUS 1998; 7(3):35-42.

25. Vidal SA, Arruda BKG, Vanderlei LC, Frias PG. Avaliação da série histórica dos nascidos vivos em unidade terciária de Pernambuco: 1991 a 2000. Rev. Assoc. M ed. Bras. 2005; 51(1):17-22.

26. Maia M AC. Caracterização dos nascidos vivos hospitalares no primeiro ano de implantação do Subsistema de Informação sobre N ascidos Vivos, em município de Minas Gerais, Brasil, 1996. Rev. Saude Publica 1997; 31(6):581-585.

27. Kale PL. Primeira Avaliação do Sistema de Informações sobre $\mathrm{N}$ ascidos Vivos no M unicípio de Niterói. Cad Saude Coletiva 1997; 5(1):53-64.

28. Bohland AK, M ello Jorge M HP. M ortalidade infantil de menores de um ano de idade na região Sudoeste do Estado de São Paulo. Rev. Saude Publica 1999; 33(4):366-373.

29. Albuquerque WA, Menezes SS, Santana HS. Análise do perfil das mães dos nascidos vivos em Carbonita, M inas Gerais no ano de 1999, pelo estudo dos dados do SINASC. Rev. Bras. Saude Mater. Infant 2001; 1(2):137-143.

30. Carniel EF, Antônio MARGM, Mota MRML, Morcillo AM, Zanolli ML. A Declaração de Nascido Vivo como orientadora de ações de saúde em nível local. Rev. Bras. Saude M ater. Infant 2003; 3(2):165174.
31. Almeida MTC. A identificação de mortes durante 0 ciclo gravídico puerperal: uma contribuição à vigilância da mortalidade materna no Estado do Rio de Janeiro [dissertação]. Rio de Janeiro (RJ): Escola Nacional de Ciências Estatísticas; 2003.

32. Sousa MH. U tilização de sistemas de informações em saúde na área de morbidade materna grave (near miss) e mortalidade materna [tese]. Campinas (SP): U niversidade Estadual de Campinas; 2006.

33. Moura ERF, Holanda Jr. F, Rodrigues MSP. Avaliação da assistência pré-natal oferecida em uma microrregião de saúde do Ceará, Brasil. Cad Saude Publica 2003; 19(6):1791-1799.

34. Gentile FP, Noronha Filho G, Cunha AA. Associação entre a remuneração da assistência de cesariana em maternidades do Rio de Janeiro: uma revisão da hipótese de Carlos Gentile de M ello. Cad Saude Publica 1997; 13(2):221-226.

35. Saraceni V, Leal MC. Avaliação da efetividade das campanhas para eliminação da sífilis congênita na redução da morbimortalidade perinatal: Município do Rio de Janeiro, 1999-2000. Cad Saude Publica 2003; 19(5):1341-1349.

36. Gomes M ASM, Lopes JMA, M oreira M EL, Gianini NOM. Assistência e mortalidade neonatal no setor público do Município do Rio de Janeiro, Brasil: uma análise do período 1994/2000. Cad Saude Publica 2005; 21(4):1269-1277.

37. Schramm JMA, Szwarcwald CL, Esteves MAP. Assistência obstétrica e risco de internação na rede de hospitais do Estado do Rio de Janeiro. Rev. Saude Publica 2002; 36(5): 590-597.

38. Campos TP, Carvalho MS. Assistência ao parto no Município do Rio de Janeiro: perfil das maternidades e 0 acesso da clientela. Cad Saude Publica 2000; 16(2):411-420.

39. Giglio MRP, Lamounier JA, Morais Neto OL. Via de parto e risco para mortalidade neonatal em Goiânia no ano de 2000. Rev. Saude Publica 2005; 39(3):350-357.

40. Costa M CO, Santos CAT, Sobrinho CL, Freitas JO, Ferreira KASL. Indicadores materno-infantis na adolescência e juventude: sociodemográfico, prénatal, parto e nascidos vivos. J pediatr. (Rio J.) 2001; $77(3): 235-242$

41. Gama SGN, Szwarcwald CL, Leal MC, Theme Filha M M. Gravidez na adolescência como fator de risco para baixo peso ao nascer no Município do Rio de Janeiro, 1996 a 1998. Rev. Saude Publica 2001; 35(1): 74-80.

42. Costa MCO, Santos CAT, Sobrinho CL, Freitas JO, Ferreira KASL, Silva MA, Paula PLB. Estudo dos partos e nascidos vivos de mães adolescentes e adultas jovens no M unicípio de Feira de Santana, Bahia, Brasil, 1998. Cad Saude Publica 2002; 18(3):715-722.

43. Gama SGN, Szwarcwald CL, Leal MC. Experiência de Gravidez na adolescência, fatores associados e resultados perinatais entre puérperas de baixa renda. Cad Saude Publica 2002; 18(1):153-161. 
44. Goldenberg P, Figueiredo MCT, Silva RS. Gravidez na adolescência, pré-natal e resultados perinatais em M ontes Claros, M inas Gerais, Brasil. Cad Saude Publica 2005; 21(4):1077-1086.

45. Gouveia N, Bremner SA, Novaes HMD. Association between ambient air pollution and birth weight in São Paulo, Brazil. J Epidemiol Community H ealth 2004; 58:11-17.

46. Costa CE, Gotlieb SLD. Estudo Epidemiológico do peso ao nascer a partir da Declaração de Nascido Vivo. Rev. Saude Publica 1998; 32(4):328-334.

47. M orais N eto OL, Barros MBA. Fatores de risco para mortalidade neonatal e pós-neonatal na Região Centro-O este do Brasil: linkage entre bancos de dados de nascidos vivos e óbitos infantis. Cad Saude Publica 2000; 16(2):477-485.

48. Weirich CF, Andrade ALSS, Turchi MD, Silva SA $M$ orais- $\mathrm{N}$ eto $\mathrm{OL}, \mathrm{M}$ inamisava $\mathrm{R}, \mathrm{M}$ arques $\mathrm{SM}$. Neonatal mortality in intensive care units of Central Brazil. Rev. Saude Publica 2005; 39(5):775-781.

49. Giglio MRP, Lamounier JA, M orais Neto OL, César CC. Baixo peso ao nascer em coorte de recémnascidos em Goiânia-Brasil no ano de 2000. Rev. Bras. Ginecol. Obstet. 2005; 27(3):130-136.

50. Freitas PF, Drachler ML, Leite JCC, Grassi PR. De sigualdade social nas taxas de cesariana em primíparas no Rio Grande do Sul. Rev. Saude Publica 2005; 39(5):761-767.

51. Goldani MZ, Barbieri MA, Bettiol H, Barbieri MR, Tomkins A. Infant mortality rates according to socioeconomic status in a Brazilian city. Rev. Saude Publica 2001; 35(3):256-261.

52. Guimarães MJB, M arquês NM, M ello Filho DA, Szawarcwald $\mathrm{CL}$. Condição de vida e mortalidade infantil: diferenciais intra-urbanos no Recife, Pernambuco, Brasil. Cad Saude Publica 2003; 19(5):14131424.

53. Goldani MZ, Benatti R, Silva AAM, Bettiol H, Correa JCW, Tietzmann M, Barbieri MA. Narrowing inequalities in infant mortality in Southern Brazil. Rev. Saude Publica 2002; 36(4):478-483.

Artigo apresentado em 08/05/2008

Aprovado em 08/05/2008

Versão final apresentada em 19/01/2009 